\title{
Phytoprotection
}

\section{Quelques enzymes végétales à potentiel antimicrobien}

\section{A. Asselin}

Volume 74, numéro 1, 1993

URI : https://id.erudit.org/iderudit/706032ar

DOI : https://doi.org/10.7202/706032ar

Aller au sommaire du numéro

\section{Éditeur(s)}

Société de protection des plantes du Québec (SPPQ)l

\section{ISSN}

0031-9511 (imprimé)

1710-1603 (numérique)

\section{Découvrir la revue}

\section{Citer cet article}

Asselin, A. (1993). Quelques enzymes végétales à potentiel antimicrobien. Phytoprotection, 74(1), 3-18. https://doi.org/10.7202/706032ar

\section{Résumé de l'article}

Cet article porte sur un certain nombre d'activités enzymatiques d'origine végétale possédant la propriété d'altérer des polysaccharides pariétaux bactériens ou fongiques. Ces activités sont présentées selon les cinq aspects suivants. Premièrement, on traitera de la détection et de la diversité électrophorétiques de certaines enzymes à potentiel antimicrobien. Ces enzymes sont les chitinases, les déacétylases de la chitine, les chitosanases, les §-1,3-glucanases, les lysozymes et d'autres activités hydrolysant les parois bactériennes ou fongiques. Deuxièmement, on présentera des résultats d'inhibition de croissance de certains microorganismes exposés à des enzymes végétales séparées électrophorétiquement. Troisièmement, certaines données sur des plants transgéniques exprimant des enzymes à potentiel antimicrobien seront discutées brièvement. Quatrièmement, quatre enzymes présentes chez les végétaux seront comparées à leur équivalent microbien ou animal. Ces enzymes sont les chitinases, les chitosanases, les ßs-1,3-glucanases (laminarinases) et les lysozymes. La comparaison indique que toutes ces activités se trouvent chez certains microorganismes et animaux supérieurs en plus de leur présence chez les végétaux. Les chitosanases n'ont cependant pas encore été rapportées chez les animaux. Il s'agit là d'une exception fort intéressante par rapport aux autres enzymes mais dont la signification n'a pas été élucidée. Enfin, le cinquième aspect traitera de quatre types d'enzymes végétales à potentiel antimicrobien en fonction des protéines végétales reliées à la pathogenèse (protéines $\mathrm{PR}$ ). Les protéines $\mathrm{PR}$ sont des protéines de stress dont la synthèse $d e$

novo est stimulée par divers agents biotiques ou abiotiques. Dans certaines conditions de stress, les chitinases, les chitosanases, les §-1,3-glucanases (laminarinases) et les lysozymes d'origine végétale sont des protéines PR. Par contre, ces activités enzymatiques peuvent être aussi exprimées de façon constitutive et sous diverses formes moléculaires dans certains organes des plantes supérieures. Les organes reproducteurs semblent particulièrement une excellente source de certaines isoformes de ces enzymes à potentiel antimicrobien.
Ce document est protégé par la loi sur le droit d'auteur. L’utilisation des services d'Érudit (y compris la reproduction) est assujettie à sa politique d'utilisation que vous pouvez consulter en ligne.

https://apropos.erudit.org/fr/usagers/politique-dutilisation/ 


\title{
Quelques enzymes végétales à potentiel antimicrobien
}

\author{
Alain Asselin ${ }^{1}$
}

Reçu 1992-11-18; accepté 1993-05-11

Cet article porte sur un certain nombre d'activités enzymatiques d'origine végétale possédant la propriété d'altérer des polysaccharides pariétaux bactériens ou fongiques. Ces activités sont présentées selon les cinq aspects suivants. Premièrement, on traitera de la détection et de la diversité électrophorétiques de certaines enzymes à potentiel antimicrobien. Ces enzymes sont les chitinases, les déacétylases de la chitine, les chitosanases, les ß-1,3-glucanases, les lysozymes et d'autres activités hydrolysant les parois bactériennes ou fongiques. Deuxièmement, on présentera des résultats d'inhibition de croissance de certains microorganismes exposés à des enzymes végétales séparées électrophorétiquement. Troisièmement, certaines données sur des plants transgéniques exprimant des enzymes à potentiel antimicrobien seront discutées brièvement. Quatrièmement, quatre enzymes présentes chez les végétaux seront comparées à leur équivalent microbien ou animal. Ces enzymes sont les chitinases, les chitosanases, les ß-1,3-glucanases (laminarinases) et les lysozymes. La comparaison indique que toutes ces activités se trouvent chez certains microorganismes et animaux supérieurs en plus de leur présence chez les végétaux. Les chitosanases n'ont cependant pas encore été rapportées chez les animaux. Il s'agit là d'une exception fort intéressante par rapport aux autres enzymes mais dont la signification n'a pas été élucidée. Enfin, le cinquième aspect traitera de quatre types d'enzymes végétales à potentiel antimicrobien en fonction des protéines végétales reliées à la pathogenèse (protéines PR). Les protéines PR sont des protéines de stress dont la synthèse de novo est stimulée par divers agents biotiques ou abiotiques. Dans certaines conditions de stress, les chitinases, les chitosanases, les ß-1,3-glucanases (laminarinases) et les lysozymes d'origine végétale sont des protéines PR. Par contre, ces activités enzymatiques peuvent être aussi exprimées de façon constitutive et sous diverses formes moléculaires dans certains organes des plantes supérieures. Les organes reproducteurs semblent particulièrement une excellente source de certaines isoformes de ces enzymes à potentiel antimicrobien.

\section{Asselin, A. 1993. Plant enzymes with antimicrobial properties. PHYTOPRO- TECTION 74: 3-18.}

Some enzymatic activities found in plants and exhibiting the capacity to alter bacterial or fungal cell wall polysaccharides are presented according to the following five aspects. The electrophoretic detection and diversity of some enzymes with antimicrobial potential will first be discussed. These enzymes are: chitinases, chitin deacetylases, chitosanases, ß-1,3-glucanases, lysozymes and some activities hydrolysing bacterial or fungal walls. Secondly, results dealing with growth inhibition assays of some microorganisms exposed to

1. Département de phytologie, Faculté des sciences de l'agriculture et de l'alimentation,

Université Laval, Québec (Québec), Canada G1K 7P4 
plant enzymes separated by electrophoresis will be considered. Thirdly, some data on transgenic plants expressing enzymes of antimicrobial potential will be briefly discussed. Four enzymes found in plants will then be compared to their microbial or animal equivalent. These enzymes are: chitinases, chitosanases, ß-1,3-glucanases (laminarinases) and lysozymes. The comparison shows that all these activities can be found in some microorganisms and higher animals in addition to their presence in plants. However, chitosanases have not been yet reported in animals. This is a very interesting exception when compared to the other enzymes, but whose significance has not been elucidated. Finally, four plant enzymes with antimicrobial potential will be presented in relation with the pathogenesis-related proteins (PR proteins). The PR proteins are stress proteins for which de novo synthesis is stimulated by various abiotic or biotic agents. Under certain stress conditions, plant chitinases, chitosanases, ß-1,3-glucanases (laminarinases) and lysozymes are PR proteins. However, these enzymatic activities can be expressed constitutively as various molecular forms in some organs of higher plants. Reproductive organs seem an excellent source of some isoforms of these potentially antimicrobial enzymes.

\section{INTRODUCTION}

Les végétaux possèdent une gamme de substances très variées pour lutter contre les divers agents pathogènes. Ces substances sont exprimées de façon constitutive ou inductive. Parmi ces substances, certaines enzymes ont la propriété d'hydrolyser des polysaccharides pariétaux bactériens ou fongiques. Ainsi, les chitinases dégradent la chitine, les chitosanases affectent le chitosane, les ß-1,3-glucanases hydrolysent les $B-1,3-$ glucanes similaires à la laminarine et les lysozymes dégradent le peptidoglycane. La chitine, le chitosane et les $\beta-1,3$-glucanes se trouvent souvent dans les parois de champignons (y compris les champignons phytopathogènes) tandis que le peptidoglycane est un constituant des parois de bactéries.

Le but de cette revue de littérature est de présenter certaines nouvelles techniques biochimiques analytiques pour l'étude et la détection de diverses enzymes à potentiel antimicrobien et d'autre part de comparer la présence de quatre enzymes végétales à potentiel antimicrobien chez d'autres organismes afin de déceler les éléments communs qui ont trait à ces enzymes dites de défense. Finalement, quatre enzymes sont présentées quant à leur réaction comme protéines reliées à la pathogenèse (protéines PR).

\section{DÉTECTION ET DIVERSITÉ ÉLECTROPHORÉTIQUES D'ENZYMES VÉGÉTALES À POTENTIEL ANTIMICROBIEN}

Cette section se limite à l'étude de six activités enzymatiques [chitinases, déacétylases de la chitine, chitosanases, ß1,3-glucanases (laminarinases), B-1,3-glucanases (activités envers le $ß-1,3$-glucane de la levure Saccharomyces) et lysozymes] en plus de considérer l'hydrolyse de parois bactériennes ou fongiques. L'approche technique est toujours la même. Les substrats sont incorporés à la matrice de polyacrylamide précédant la séparation électrophorétique des protéines. Après l'électrophorèse, l'activité enzymatique altérant le substrat incorporé au gel est révélée par des techniques de coloration différentielle du substrat intact comparé au substrat altéré ou par la visualisation de la perte d'opacité du substrat. Les techniques sont présentées de façon succincte.

\section{Les chitinases}

Les chitinases regroupent diverses activités hydrolysant la chitine. La chitine est un polymère linéaire de $\mathrm{B}-1,4-\mathrm{N}$-acétylD-glucosamine qui est l'aminopolysaccharide le plus abondant dans la nature, avec une production annuelle estimée à $150000 \mathrm{t}$ (Knorr 1986). La chitine est présente dans l'exosquelette de plusieurs 
invertébrés marins (crustacés), dans la cuticule des insectes et dans les parois et septa de la plupart des champignons (Cabib et al. 1982). La chitine est une structure idéalisée parce qu'il peut exister jusqu'à un résidu de la D-glucosamine (résidu déacétylé) dans une séquence de six résidus de la N-acétyl-D-glucosamine (Blackwell 1988).

Il existe plusieurs formes cristallines et de nombreux dérivés chimiques de ce polysaccharide (Pont Lezica et QuesadaAllué 1990). Parmi ces dérivés, la glycol chitine est une forme de chitine soluble dans I'eau (Hirano 1988) qui peut servir de substrat à diverses activités chitinolytiques (Koga et Kramer 1983). À partir de ces observations, nous avons utilisé la glycol chitine comme substrat incorporé à un gel de polyacrylamide pour la détection des chitinases après une séparation électrophorétique en condition native ou dénaturante (Trudel et Asselin 1989). L'avantage de cette approche est la capacité de déceler rapidement la diversité des formes électrophorétiques des chitinases. C'est ainsi qu'un extrait foliaire de tabac (Nicotiana tabacum L. cv. Xanthinc) infecté par le virus de la mosaïque du tabac contient jusqu'à 13 formes moléculaires différentes ayant une activité chitinolytique. Deux systèmes électrophorétiques (à pH 8,9 et 4,3) en condition native sont utilisés pour déceler la diversité de ces formes (Trudel et al. 1989). Les chitinases peuvent être aussi séparées dans des gels bidimensionnels. Par exemple, après une séparation en condition native, les chitinases peuvent être séparées dans une seconde dimension selon leur masse moléculaire apparente à l'aide du sodium de dodécyl sulfate (SDS) (Trudel et al. 1989). Les chitinases végétales ont une masse moléculaire apparente comprise entre 25 et $30 \mathrm{kD}$ (Trudel et al. 1989). II s'agit de masses moléculaires apparentes puisque la dénaturation est partielle parce qu'elle ne se fait pas en présence d'agents réducteurs (tels le mercaptoéthanol ou le dithiothréitol) à cause de l'inactivation de la plupart des chitinases à la suite d'une dénaturation en présence d'agents réducteurs (Trudel et Asselin 1989; Trudel et al. 1989).

Les avantages de la détection électrophorétique des chitinases sont nombreux.
La technique est simple, rapide, sensible et compatible avec les divers systèmes électrophorétiques couramment utilisés (Trudel et Asselin 1989). Une image d'ensemble des activités chitinasiques peut être visualisée lors de comparaisons d'extraits d'organes ou de tissus en situation physiologique normale (EI Ghaouth et al. 1991; Majeau et al. 1990) ou lors de la réaction des végétaux à divers types de stress (El Ghaouth et al. 1992; Trudel et al. 1989). La technique présente aussi certaines lacunes. D'une part, il est possible que certaines chitinases n'agissent que sur la chitine cristalline. II faudrait donc utiliser comme substrat de la chitine insoluble par rapport à la glycol chitine soluble normalement utilisée (Trudel et Asselin 1989). D'autre part, il est probable aussi que des activités enzymatiques puissent être inactivées par certaines conditions reliées à la préparation des échantillons pour l'électrophorèse. Nous avons déjà mentionné la sensibilité de certaines enzymes aux agents réducteurs.

Malgré ces inconvénients, cette approche a permis de mieux étudier la diversité des formes moléculaires des chitinases végétales (El Ghaouth et al. 1992; Majeau et al. 1990; Trudel et al. 1989). Un des résultats intéressants est certes la présence d'une forme de chitinase spécifique à un organe végétal précis. Ainsi, une forme de chitinase spécifique aux racines de concombre (Cucumis sativus L.) a été récemment rapportée (Majeau et al. 1990). La signification de la diversité des formes moléculaires de chitinases en fonction des organes est encore inconnue. On présume toujours que les chitinases végétales agissent sur des substrats exogènes. II ne faut pas cependant réfuter trop hâtivement la possibilité d'interactions de chitinases avec des composés endogènes aux végétaux (Benhamou et Asselin 1989). Soulignons enfin que la technique électrophorétique a été utilisée récemment pour l'étude des interactions entre plantes et mycorhizes (Dumas-Gaudot et al. 1992a, 1992b).

\section{Les déacétylases de la chitine}

Les déacétylases de la chitine enlèvent le groupement $\mathrm{N}$-acétylé des résidus polymérisés de la $\mathrm{N}$-acétyl-D-glucosamine de la chitine. Ce faisant, la chitine peut être 
convertie ultimement en chitosane (polymère de la $B$-1,4-D-glucosamine) si la déacétylation est complète. Le chitosane a des propriétés bien différentes de celles de la chitine. Par exemple, il est soluble en milieu acide. Des déacétylases de la chitine ont été rapportées dans des extraits de champignons (Araki et Ito 1988; Kauss et Bauch 1988) et de certains invertébrés. Ces déacétylases pourraient théoriquement posséder un potentiel antimicrobien puisqu'elles peuvent altérer l'intégrité de la chitine.

Une méthode de détection électrophorétique des activités des déacétylases de la chitine a été mise au point en adaptant une approche analogue à la détection des chitinases et en se servant de deux propriétés différenciant la chitine et le chitosane. Premièrement, le chitosane est sensible à la dégradation chimique par l'acide nitreux et a une affinité plus grande que la chitine pour un fluorochrome, le Calcofluor White M2R ${ }^{\circledast}$ (Sigma, StLouis, MO, U.S.A.) (Trudel et Asselin 1990). En utilisant ces propriétés différentielles, il est possible de déceler électrophorétiquement en condition native ou dénaturante diverses formes moléculaires des déacétylases de la chitine (Trudel et Asselin 1990). Les champignons Zygomycètes produisent abondamment de telles enzymes. Au moins deux formes peuvent être décelées en condition native et quatre niveaux de masses moléculaires apparentes sont aussi observés en condition dénaturante (Trudel et Asselin 1990). Jusqu'à présent, aucune déacétylase de la chitine n'a pu être détectée chez les végétaux ( $\mathrm{J}$. Trudel et $A$. Asselin, données non publiées). De plus, les déacétylases de la chitine de certains Zygomycètes ne se sont pas avérées antifongiques lors d'essais d'inhibition de croissance fongique (J. Trudel et A. Asselin, données non publiées).

\section{Les chitosanases}

Le chitosane est un terme générique représentant différents états de déacétylation de la chitine. Le chitosane pur (c'està-dire complètement déacétylé) est difficile à obtenir. En général, les chitosanes correspondent à des populations plus ou moins homogènes de polymères. Le degré de déacétylation (Nanjo et al. 1991) et de polymérisation des résidus doit être spécifié pour bien comparer les préparations de chitosanes entre elles. Le chitosane est généralement préparé chimiquement par la déacétylation alcaline de la chitine. Cependant, le chitosane se trouve dans les parois de certains champignons (les Zygomycètes, par exemple) (Novaes-Ledieu et Garcia-Mendoza 1981). L'étude électrophorétique des chitosanases végétales est également possible comme pour les activités chitinolytiques et selon les mêmes principes de séparation et de détection (Grenier et Asselin 1990). Chez les feuilles d'orge (Hordeum vulgare L.) stressées, quatre formes de chitosanases acidiques et deux formes basiques ont été décelées (Grenier et Asselin 1990). Chez les cotylédons de concombre stressé, quatre chitosanases acidiques sont présentes. Toutes les masses moléculaires apparentes sont entre 10 et 24 kD (Grenier et Asselin 1990). Le nombre de formes moléculaires de chitosanases semble inférieur à celui des chitinases.

Un inventaire des activités de chitosanases a été effectué chez plusieurs végétaux où les graines constituent une source importante de chitosanases (El Ouakfaoui et Asselin 1992a). Des chitosanases spécifiques à certains organes de concombre ont été aussi observées (EI Ouakfaoui et Asselin 1992b). Comme pour les chitinases végétales, on prend pour acquis dans la majorité des publications sur les chitosanases que les substrats sont $d$ 'origine exogène aux végétaux. Même s'il n'existe pas de données précises à ce sujet, les chitosanases agissent peut-être aussi sur des composés végétaux (El Ouakfaoui et Asselin 1992a, 1992b). Enfin, il est intéressant de noter que le chitosane peut être analysé et caractérisé électrophorétiquement. Par cette nouvelle approche, les produits d'hydrolyse du chitosane peuvent être aussi étudiés (Audy et Asselin 1992).

Les ß-1,3-glucanases (laminarinases)

Les ß-1,3-glucanes sont des constituants fréquents des parois fongiques. L.'hydrolyse de ces produits peut être reliée à des effets antifongiques (Mauch et al. 1988a, 1988b). La diversité des formes moléculaires des ß-1,3-glucanases hydrolysant la laminarine (oligomère de $\beta$-1,3-glucose, soluble dans l'eau) comme substrat 
modèle peut être facilement analysée électrophorétiquement. Après la séparation et l'incubation avec la laminarine incorporée au gel de séparation ou dans un gel de contact, les bandes de lyse sont révélées par une coloration de la laminarine avec le bleu d'aniline (Côté et al. 1989) ou le sirofluor (Côté et al. 1991a, 1991b). Les bandes de lyse peuvent correspondre à des signaux sans fluorescence ou de fluorescence plus prononcée par rapport à la fluorescence du substrat intact qu'est la laminarine. Les diverses formes moléculaires de ces hydrolases déjà connues et révélées électrophorétiquement (Côté et al. 1989) comprennent des protéines PR (protéines reliées à la pathogenèse) du tabac.

La méthode électrophorétique permet donc de déceler les activités ß-1,3-glucanasiques qui précédemment étaient caractérisées biochimiquement et sérologiquement. De plus, certaines laminarinases sont retardées dans leur migration életrophorétique en condition native. II $\mathrm{s}^{\prime}$ agit donc de la possibilité supplémentaire d'utiliser une certaine forme d'électrophorèse d'affinité (Côté et al. 1989). Un désavantage potentiel à I'utilisation de la laminarine provient de son origine, c'est-à-dire qu'il s'agit d'un polysaccharide de réserve extrait d'algues (le plus souvent de Laminaria spp.). Les ß-1,3glucanes des parois fongiques sont différents si l'on considère le degré de polymérisation et leur structure secondaire et tertiaire. C'est pourquoi des $\beta-1,3-$ glucanes plus représentatifs des $ß-1,3-$ glucanes constituants fongiques pourraient être plus avantageux comme substrats que la laminarine.

\section{Les ß-1,3-glucanases actives envers les ß-1,3-glucanes de la levure Saccharomyces}

Récemment, la laminarine a été remplacée comme substrat modèle des $\beta-1,3-$ glucanases par une préparation de $ß-1,3-$ glucane de levure (Saccharomyces cerevisiae Meyen ex Hansen). Ce composé est relativement purifié (environ $88 \%$ de résidus de $\beta-1,3$-glucose) et peut être incorporé au gel de polyacrylamide grâce à sa solubilité à pH alcalin (Cabib et Bowers 1971). II démontre deux avantages comparativement à la laminarine. Premièrement, la coloration au bleu d'ani- line ou au sirofluor ressort mieux en photographie grâce au contraste accru entre le substrat intact et hydrolysé. Deuxièment, les gels avec le glucane de levure peuvent se conserver beaucoup plus longtemps que ceux imprégnés de laminarine (J. Grenier et A. Asselin, données non publiées). La raison en est fort simple : la laminarine s'élue très rapidement de la matrice du gel même à une concentration élevée d'acrylamide $115 \%$, $\mathrm{p} / \mathrm{vol}$, par exemple). II est donc utile de remplacer dans la mesure du possible la laminarine par les ß-1,3-glucanes fongiques.

\section{Les lysozymes}

Les lysozymes se définissent comme les hydrolases actives envers le peptidoglycane des parois du Micrococcus luteus (Schroeter) Conn (synonyme: M. Iysodeikticus Fleming). Biochimiquement, les lysozymes sont des $\mathrm{N}$-acétylmuramoylhydrolases du peptidoglycane (Jollès et Jollès 1984). Elles hydrolysent donc un des polymères structuraux des parois bactériennes. II est possible d'étudier électrophorétiquement la diversité des lysozymes d'origine végétale en incorporant au gel de polyacrylamide une suspension de cellules du $M$. luteus ou en utilisant un gel-contact contenant la même suspension de cellules bactériennes. Techniquement, les méthodes électrophorétiques sont simples à utiliser et relativement sensibles quant à la capacité de détection de l'activité lysozymale. En condition dénaturante, on peut déceler moins d'un picogramme d'équivalent du lysozyme de blanc d'oeuf de poule après une incubation de $18 \mathrm{~h}$ (Audy et al. 1989).

La diversité électrophorétique des lysozymes a été étudiée dans plusieurs végétaux (Audy et al. 1988a, 1988b; Majeau et al. 1990; Trudel et al. 1989). Un inventaire des formes électrophorétiques lysozymales a aussi été complété récemment (Audy et al. 1990). Les lysozymes végétaux peuvent présenter jusqu'à cinq formes électrophorétiques différentes dans un même organe sain. La détection des activités lysozymales en utilisant les suspensions du Micrococcus à la place du peptidoglycane bactérien purifié présente cependant des désavantages du fait qu'une activité lytique envers le 
Micrococcus n'est pas nécessairement un lysozyme au sens strict. Il peut s'agir alors d'amidases, de glucosaminidases ou même d'autres hydrolases (Béliveau et al. 1991). L'identification des formes lysozymales basée seulement sur la lyse en gel du $M$. luteus exige donc de la prudence.

\section{La lyse des parois bactériennes, de parois et de spores fongiques}

En relation avec la section précédente sur les lysozymes, diverses parois bactériennes (autres que celle du $M$. luteus) peuvent être incorporées à des gels de polyacrylamide dans le but d'étudier des enzymes à potentiel antimicrobien. Cette méthodologie peut s'appliquer d'abord et avant tout aux bactéries de type Gram positif (Leclerc et Asselin 1989; Potvin et al. 1988).

En plus de pouvoir visualiser en microscopie électronique la lyse de parois fongiques (Benhamou et al. 1989), les altérations de parois fongiques peuvent être étudiées facilement après une séparation électrophorétique en gels de polyacrylamide. Comme pour les parois bactériennes, la disparition de l'opacité, liée aux parois fongiques incorporées dans la matrice des gels de polyacrylamide, sert d'indice visuel de I'hydrolyse du substrat pour déceler et photographier les zones de lyse par l'illumination indirecte sur fond noir (Potvin et al. 1988). Selon notre expérience, les parois de Zygomycètes (Mucor spp., Rhizopus spp.), Ascomycètes (Verticillium spp., Fusarium spp., Saccharomyces spp., Schizosaccharomyces spp.), Basidiomycètes (Agaricus spp.) et Phycomycètes (Phytophthora spp.) peuvent être facilement soumises à la méthode électrophorétique (J. Grenier et $\mathrm{A}$. Asselin, données non publiées). Les spores de plusieurs champignons peuvent aussi être incorporées aux gels de polyacrylamide dans le but d'étudier les facteurs lytiques qui les affectent. Des activités Iytiques envers les spores de certains champignons Ascomycètes ont été étudiées par le biais de l'électrophorèse uni- ou bidimensionnelle (Grenier et Asselin 1990).

\section{ESSAIS D'INHIBITION DE CROISSANCE MICROBIEINNE APRESS SÉPARATION ÉLECTROPHORÉTIQUE DE PROTÉINES}

La section précédente concernait une caractérisation électrophorétique de certaines enzymes à potentiel antimicrobien par le biais de l'utilisation de substrats qui ne correspond pas nécessairement à la capacité réelle d'inhibition de la croissance microbienne active par ces mêmes enzymes. Dans la présente section, l'inhibition de croissance signifie tout simplement un effet décelable visible d'une enzyme sur la croissance normale microbienne. On ne veut pas traiter ici des divers mécanismes d'inhibition qu'ils soient de nature biocide (létale) ou biostatique. En général et de façon usuelle, l'effet inhibiteur antimicrobien des protéines végétales (en particulier les chitinases et les ß-1,3-glucanases) a été étudié en utilisant des protéines purifiées (Mauch et al. 1988a, 1988b; Schlumbaum et al. 1986), évaluées en plaques d'agar ou d'agarose. Par cette approche, des chitinases et des $ß$-1,3-glucanases végétales ont été reconnues comme des enzymes à potentiel antimicrobien parce qu'elles arrêtent, surtout en synergie, le développement de la croissance active des microorganismes. Évidemment, ces essais nécessitent la purification des enzymes.

Une approche alternative électrophorétique a été utilisée récemment pour analyser l'effet inhibiteur de certaines protéines végétales après leur séparation électrophorétique. Dans un premier cas, les protéines (incluant une chitinase) ont été séparées par une électrophorèse en gel de polyacrylamide dans des conditions natives et $0,5 \%(p / v o l) d^{\prime}$ agar nutritif contenant une suspension de spores $\left(2 \times 10^{5} \mathrm{~mL}^{-1}\right)$ a été déposé sur la matrice de polyacrylamide. Après une incubation de $48 \mathrm{~h}$, les zones d'inhibition de la croissance fongique liée à une chitinase basique de tabac ont été décelées visuellement (De Bolle et al. 1991). Dans un second cas, les protéines de graines de diverses plantes ont été séparées électrophorétiquement en gel de polyacrylamide dans des conditions dénaturantes 
partielles (SDS) et renaturées en présence de Triton X-100 (Vigers et al. 1991) selon une méthodologie identique à celle de Potvin et al. (1988). Dans ce cas, cependant, l'inhibition efficace de la croissance du champignon Candida spp. a requis la présence d'un fongicide chimique du type de la nikkomycine.

Nous avons évalué l'effet de toute une gamme d'enzymes végétales, animales et microbiennes sur la croissance active du Clavibacter michiganensis (Smith) Davis (bactérie Gram positive, agent du flétrissement bactérien de la pomme de terre), du Verticillium dahliae Klebahn (champignon Ascomycète, agent du flétrissement de la pomme de terre) et du Fusarium oxysporum f. sp. radicis-lycopersici Jarvis et Shoemaker (champignon Ascomycète, agent du flétrissement de la tomate). Les résultats montrent que le lysozyme de blanc d'oeuf de poule (HEWL) est supérieur à toutes les autres enzymes testées pour inhiber de façon efficace le développement de ces trois agents pathogènes ( $\mathrm{J}$. Trudel et $\mathrm{A}$. Asselin, données non publiées). II suffit d'à peine $100 \mathrm{ng}$ de HEWL en gel de polyacrylamide pour causer l'inhibition. Évidemment, le HEWL inhibe le Clavibacter par son activité lysozymale alors que son activité chitinasique explique l'arrêt de croissance du Fusarium et du Verticillium (J. Trudel et A. Asselin, données non publiées). Cette nouvelle approche nous indique finalement qu'il est possible d'obtenir des inhibitions efficaces par des enzymes seules sans devoir recourir à des effets de synergie (Mauch et al. 1988a, 1988b).

\section{ÉVALUATION DU POTENTIEL ANTIMICROBIEN PAR LES PLANTS TRANSGÉNIQUES}

\section{Les modèles existants}

Malgré les nouvelles techniques électrophorétiques et les nombreuses données biochimiques et ultrastructurales, un potentiel antimicrobien doit être ultimement éprouvé par le biais de plants transgéniques exprimant une protéine fonctionnelle et localisée correctement. II existe déjà plusieurs publications présentant des résultats fort variables quant à l'expression efficace de l'activité antimicrobienne. Malgré un niveau d'expression très élevé d'une chitinase, des plants transgéniques de Nicotiana spp. n'ont pu démontrer une résistance ou tolérance accrue à des pathogènes (Neuhaus et al. 1991a). II faut distinguer la présence des chitinases vacuolaires et extracellulaires (Samac et Shah 1991) et réaliser que les plants transgéniques doivent exprimer ces enzymes selon leur localisation correcte (Neuhaus et al. 1991b). La nécessité de signaux peptidiques pour la sécrétion efficace d'enzymes à potentiel antimicrobien doit être prise en considération (Lund et Dunsmuir 1992).

Même si des plants transgéniques démontrent un niveau accru d'activité antifongique (Broglie et al. 1991), il n'est pas évident que cette approche soit sans faille ou sans danger. D'une part, il est bien connu que les microorganismes peuvent souvent développer des souches ou isolats résistants à des facteurs antimicrobiens. En principe, ce phénomène est certainement concevable aussi avec les enzymes lytiques antimicrobiennes. D'autre part, connaissant cette facilité microbienne d'adaptation, il est peut-être plus sage d'envisager l'utilisation de la résistance induite systémique et généralisée. Une meilleure connaissance de l'activité coordonnée des divers facteurs antimicrobiens lors de la réponse de cette résistance induite systémique mérite une attention particulière (Ward et al. 1991).

\section{Le modèle tabac-HEWL}

Nous avons récemment produit des plants transgéniques de tabac (Nicotiana tabacum cv. Xanthi-nc) exprimant le HEWL. Cette protéine est sécrétée (Trudel et al. 1992) par l'intermédiaire de son propre peptide de signal et est active dans tous les organes testés. La protéine est produite de façon constitutive sous le contrôle du promoteur du virus de la mosaïque du chou-fleur. Les plants transgéniques exprimant le HEWL pourraient servir de modèles à bien des points de vue puisque cette protéine est l'hydrolase antimicrobienne la plus étudiée du point de vue structural, moléculaire, génétique et antimicrobien (Ibrahim et al. 1991). 


\section{PRÉSENCE D’ENZYMES À POTENTIEL ANTIMICROBIEN CHEZ D'AUTRES ORGANISMES}

\section{Les chitinases}

Chez les bactéries du type Gram négatif, les chitinases du Serratia marcescens Bizio les mieux connues (Monreal et Reese 1968) comprennent au moins cinq protéines chitinolytiques avec des masses moléculaires variant de 21 à $57 \mathrm{kD}$ (Fuchs et al. 1986). Un gène codant pour une chitinase du Serratia marcescens a été évalué pour une forme de lutte biologique envers certains champignons phytopathogènes (Fusarium oxysporum f. sp. redolens (Wollenweber) Gordon, Rhizoctonia solani Kühn et Sclerotium rolfsii Saccardo) (Shapira et al. 1989; Sundheim et al. 1988). Des plants de tabac transgéniques pour le gène chi $A$ du Serratia ont exprimé l'activité chitinasique (Lund et al. 1989; Nagel et al. 1990). L'activité principale de chitinase chez le S. marcescens est une exochitinase de masse moléculaire plus élevée que bon nombre de chitinases végétales qui agissent aussi surtout comme des endochitinases.

En plus du Serratia, les bactéries chitinolytiques les mieux connues proviennent des espèces d'Aeromonas, de Bacillus, de Chromobacterium, de Myxobacter et de Vibrio (Cody 1989). Pour les Actinomycètes, les chitinases du Streptomyces spp. sont les mieux caractérisées (Kamei et al. 1989; Robbins et al. 1988). En résumé, les chitinases bactériennes sont souvent des exochitinases de masse moléculaire élevée qui semblent être sécrétées dans les écosystèmes pour assurer le catabolisme de la chitine. Le potentiel antifongique de ces enzymes est à investiguer encore plus en détail en tenant compte de la stabilité de ces enzymes et de leur capacité inhibitrice.

L'autolyse des parois fongiques par le biais de chitinases est un phénomène bien connu (Reyes et al. 1988). De plus, certains cas de mycoparasitisme par des champignons semblent révéler la sécrétion de chitinases (Srivastava et al. 1985). Les chitinases fongiques ont été aussi étudiées chez les champignons entomo- phages (St-Leger et al. 1986a, 1986b), mycoparasites (Manocha et Balusubramanian 1988) et parasites de nématodes (Dackman et al. 1989).

Des activités chitinolytiques ont été rapportées chez les insectes, comme le Tribolium castaneum (Hbst.)[Coleoptera: Tenebrionidae] et le Stomoxys calcitrans (L.) [Diptera: Muscidae] (Chen et al. 1982; Kramer et Aoki 1987). II en est de même pour des chitinases d'invertébrés marins (Bjarnov 1972; Smucker et Wright 1986) et des organismes aussi divers que l'escargot (Helix pomatia L) (Takeda et al. 1966), les nématodes Ascaris spp. (Ward et Fairbairn 1972), la pieuvre (Eledone cirrhosa Lamarck) (Grisley et Boyle 1990) et les crevettes Artemia spp. (Funke et Spindler 1989). II semble que certaines chitinases d'insectes pourraient peut-être inhiber la germination de sclérotes de certains champignons phytopathogènes (Anas et al. 1989). Des données supplémentaires sont cependant nécessaires à ce sujet.

Les chitinases sont présentes tarit chez les vertébrés inférieurs (Michia et al. 1973) que chez les vertébrés supérieurs incluant le singe et I'homme (Den Tandt et al. 1988). Les chitinases semblent abondantes dans certains tissus sécréteurs des amphibiens et des poissons (Kono et al. 1990). De plus, on trouve des chitinases dans le sang des poissons (Fänge et al. 1976) et dans le sérum des chèvres (Lundblad et al. 1974).

II existe plusieurs revues de littérature sur le sujet des chitinases végétales (Boller 1988, par exemple). En comparant diverses chitinases de végétaux supérieurs, il est apparu que l'on peut distinguer au moins trois types de chitinases végétales. Les chitinases de la classe I sont des chitinases basiques avec un domaine riche en cystéine à l'extrémité $\mathrm{N}$-terminale. Les chitinases de la classe II sont similaires à celles de la classe I sauf pour l'absence du domaine riche en cystéine. Enfin, les chitinases de la classe III sont différentes en homologie de séquences des deux classes de chitinases végétales (Shinshi et al. 1990). Le domaine riche en cystéine (domaine d'environ 40 acides aminés) a été retrouvé aussi chez I'hévéine du latex de l'Hevea brasiliensis 
(Willd. ex A. Juss.) Müll., et chez des protéines de stress de la pomme de terre (Solanum tuberosum L.) (Lucas et al. 1985; Stanford et al. 1989). En résumé, les chitinases végétales ont des masses moléculaires entre 24 et $40 \mathrm{kD}$ et correspondent à au moins trois types de gènes au point de vue de la structure générale.

\section{Les chitosanases}

Jusqu'à présent, les chitosanases n'ont été rapportées que chez des bactéries, des champignons et des plantes. Plusieurs bactéries (incluant les Actinomycètes) peuvent produire de façon inductive des chitosanases (Ohtakara et al. 1988; Pelletier et Sygusch 1990). Les chitosanases microbiennes ont des masses moléculaires entre 22 et $43 \mathrm{kD}$.

Chez les champignons, les chitosanases se trouvent chez les Aspergillus, Penicillium, Rhizopus, Trichoderma et Zygorrhynchus spp. (Monaghan et al. 1973). Une chitosanase du Penicillium islandicum Sopp. a été étudiée en détail (Fenton et Eveleigh 1981) alors que des chitosanases ont été observées lors de I'autolyse du Mucor rouxii (Calmette) Wehmer (Reyes et al. 1985). Enfin, une activité chitosanasique a été observée chez le Metarhizium anisopliae (Metchnikoff) Sorokin, un champignon entomophage (St-Leger et al. 1986b).

II n'y a aucune chitosanase d'origine animale connue à ce jour. Utilisant une technique électrophorétique pour la détection des chitosanases, nous n'avons pu déceler des chitosanases dans le sérum de divers animaux (incluant l'homme) alors que des chitosanases bactériennes, fongiques ou végétales ont été facilement observées ( $\mathrm{J}$. Grenier et A. Asselin, données non publiées).

La présence des chitosanases végétales a déjà été discutée mais il suffit de souligner que les végétaux se rapprochent davantage des microorganismes (bactériens ou fongiques) en ce qui a trait aux chitosanases. On pourrait même supposer que les chitosanases végétales possèdent des homologies de structure et de fonction avec les chitosanases fongiques.

\section{Les ß-1,3-glucanases (laminarinases)} L'hydrolyse des ß-1,3-glucanes est souvent évaluée par le biais de l'hydrolyse de la laminarine. La discussion suivante ne considérera que les laminarinases au sens strict du terme. Elle exclut donc diverses $\beta$-1,3-glucanases qui sont spécifiquement actives envers des $\beta-1,3-$ glucanes insolubles.

Un nombre plutôt limité de travaux concerne les laminarinases bactériennes. Des laminarinases ont été caractérisées chez diverses bactéries (Chesters et Bull 1963) incluant les Cellvibrio spp. (Sakellaris et al. 1990), les Cytophaga spp. (Flavobacterium) (Manners et Wilson 1973), les Arthrobacterspp. (Kitamura et al. 1971) et les Bacillus spp. (Rombouts et Phaff 1976).

Plusieurs formes de laminarisases d'origine fongique ont été rapportées (Hien et Fleet 1983) surtout chez des levures, mais fait important à noter, elles n'ont pas toutes la même activité envers les substrats insolubles. Une laminarinase du type exoglucanase de la levure Saccharomyces cerevisiae a été clonée et séquencée (Klebl et Tanner 1989). Cette protéine de $29 \mathrm{kD}$ de masse moléculaire a des propriétés de lectine envers la chitine et les ß-1,3-glucanes. De telles caractéristiques $n^{\prime}$ ont pas encore été rapportées chez d'autres laminarinases.

Les laminarinases animales se trouvent chez divers invertébrés comme certains gastropodes (Cutler et Yellowlees 1979) et dans le jus digestif de l'escargot, Helix pomatia (Marshall et Grand 1975). Une exolaminarinase présente dans les oeufs non fertilisés d'oursins, Strengylocentrotus spp. (Truschel et al. 1986) a été caractérisée.

Chez les plantes, il existe divers ß-1,3glucanes de réserve ou de protection, comme la callose (Bull et Chesters 1966). Diverses endo- et exolaminarinases ont été caractérisées tant chez les monocotyles (Ballance et Svendsen 1988) que chez les dicotyles (Wong et Maclachlan 1980). Notons qu'une exo-1,3-ß-D-glucanase est intimement liée à la paroi végétale (Liénart et al. 1986). Ceci semble suggérer un rôle au niveau du métabolisme pariétal. Un certain nombre de laminarinases végétales se trouvent dans le mileu extracellulaire (Fink et al. 1988). On ne peut pas encore différencier le rôle endogène du rôle exogène de ces laminarinases. 


\section{Les lysozymes}

Les lysozymes sont des enzymes fort étudiées tant au point de vue biochimique que génétique (Jollès et Jollès 1984). Comme il existe des centaines de références sur les lysozymes, quelques références spécifiques seront présentées pour orienter une connaissance plus approfondie des diverses formes de lysozyme.

Certains lysozymes de bactériophages ont la propriété de faciliter la lyse interne des bactéries infectées. Par exemple, le bactériophage T4 a la capacité de lyser le peptidoglycane de la bactérie Escherichia coli (Migula) Castellani et Chalmers. Plusieurs de ces lysozymes ont été clonés, séquencés et exprimés dans l'E. coli (Perry et Whetzel 1984; Saedi et al. 1987). Plusieurs manipulations génétiques ont déjà été effectuées avec le phage T4, incluant l'introduction de ponts disulfures dans le but de mieux stabiliser la protéine lytique (Perry et Whetzel 1986). II y a aussi des essais pour exprimer le bactériophage T4 dans des plants de tabac transgénique (Hippe et al. 1989).

Certaines autolysines bactériennes peuvent être des lysozymes. À titre $\mathrm{d}^{\prime}$ exemple, mentionnons un lysozyme ( $\mathrm{N}$ acétylmuramoylhydrolase) du Streptococcus faecium Orla-jensen (Kawamura et Shockman 1983). Contrairement aux autres lysozymes connus, cette enzyme a une masse moléculaire relativement élevée (environ $130 \mathrm{kD}$ pour la forme inactive et environ $87 \mathrm{kD}$ pour la forme active) et est une glycoprotéine. Chez les champignons, le lysozyme des Chalaropsis spp. est bien caractérisé (Lyne et al. 1990).

Le lysozyme des insectes le mieux caractérisé est celui du Hyalophora cecropia (F.) (Boman et al. 1991). Ce lysozyme $(13 \mathrm{kD})$ est très homologue au lysozyme animal de type $c$ dont le représentant type est le HEWL. Un lysozyme similaire a été retrouvé dans le Manduca sexta (L.) [Lepidoptera: Sphingidae] (Spies et al. 1986), l'Aedes aegyptii (L.) [Diptera: Culicidae] (Rossignol et Lueders 1986), le Gryllus bimaculatus (De Geer) [Orthoptera: Gryllinae] (Schneider 1985) et divers autres insectes (Jollès et al. 1979).
Des lysozymes ont été rapportés chez des amibes (Upadhyay et al. 1977), des bivalves (McHenery et al. 1986) et d'autres invertébrés (Fenouil et Roch 1991; Jollès et Jollès 1975; Steinert et Pickwell 1984). Chez les poissons, des lysozymes de type $c$ ont été récemment séquencés et ont démontré des homologies avec les lysozymes du même type chez les oiseaux et les mammifères (Grinde et al. 1988). Avec la possibilité de produire des poissons transgéniques, les lysozymes de certains poissons pourraient présenter des avantages biotechnologiques (Grinde 1989) en aquiculture et en zootechnie.

II existe deux types de lysozymes chez les vertébrés: les lysozymes de type c (le représentant type est le HEWL et ceux de type $\mathrm{g}$ [le représentant type est le lysozyme du blanc d'oeuf de l'oie (GEWL)]. Le $H E W L$ et son équivalent humain sont des lysozymes bien caractérisés. L'expression de ces lysozymes a été étudiée dans divers systèmes hétérologues (Taniyama et al. 1991). Le gène du HEWL a été exprimé dans des plants de tabac transgénique et l'expression de la protéine sécrétée représente une activité très facile à déceler au point de vue enzymatique.

Jusqu'à présent, les lysozymes des plantes ont montré primordialement des activités chitinolytiques. Ils se distinguent donc de la plupart des autres lysozymes qui démontrent des activités lysozymales plus fortes que les activités chitinolytiques. Dans certains cas, des lysozymes (tels le lysozyme du phage T4 et le GEWL) ne démontrent que des activités lysozymales (Jollès et Jollès 1984). Les plantes ne semblent donc pas avoir le même arsenal de lysozymes que les autres organismes et elles semblent utiliser de façon préférentielle des enzymes à double fonction (chitinase/lysozyme).

\section{DES HYDROLASES SE COMPORTANT COMME DES PROTÉINES PR}

Les quatre types d'hydrolases décrites dans la section précédente seront maintenant brièvement présentés sous I'angle de leur biosynthèse accrue en réponse à divers stress chez les végétaux. Ces hydrolases peuvent, en effet, se compor- 
ter comme des protéines reliées à la pathogenèse (protéines PR), par leur accumulation et leur stimulation dans divers tissus végétaux à la suite de stress $d^{\prime}$ origine abiotique ou biotique. Plusieurs revues de littérature récentes discutent abondamment des protéines PR chez les végétaux (Bol et al. 1990; Lotan et Fluhr 1990; White et Antoniw 1991).

\section{Les chitinases}

Des protéines PR du tabac ont été les premières identifiées à des chitinases (Legrand et al. 1987). Depuis, des dizaines de chitinases ont été identifiées à des protéines PR tant chez les monocotyles que chez les dicotyles. Cependant, depuis quelques années, on observe la présence de chitinases du type PR dans divers organes floraux indemnes (Lotan et al. 1989). Il est donc devenu évident que l'accumulation de chitinases de type PR est aussi sous le contrôle du développement normal de certains organes végétaux. La réponse aux stress n'est donc qu'une facette du contrôle et de la régulation de l'expression de ces gènes.

\section{Les chitosanases}

Les chitosanases ont été identifiées comme protéines PR chez l'orge, la tomate (Lycopersicon esculentum Mill.) et le concombre (Grenier et Asselin 1990); elles sont distinctes des chitinases par leur masse moléculaire et leur spécificité de substrat. Comme pour les chitinases, on suppose que le substrat des chitosanases est un substrat exogène d'origine microbienne. Cependant, ces enzymes pourraient aussi avoir des fonctions endogènes (El Ouakfaoui et Asselin 1992a, 1992b).

\section{Les laminarinases}

Des laminarinases de tabac ont d'abord été identifiées comme protéines PR (Kauffmann et al. 1987). Comme pour les chitinases, les laminarinases semblent aussi être présentes de façon constitutive dans certains organes reproducteurs (Lotan et al. 1989). Le rôle précis des laminarinases est inconnu, mais il faut souligner la présence de divers ß-1,3glucanes (callose) chez les cellules végétales. Les laminarinases pourraient être reliées au métabolisme des composés callosiques.

\section{Les lysozymes}

Les lysozymes végétaux n'étant en fait que des activités secondaires ou complémentaires de chitinases, les lysozymes végétaux sont donc traités comme des chitinases végétales. Les remarques concernant les lysozymes s'appliquent aux chitinases comme protéines PR.

\section{CONCLUSION}

Cette revue de littérature a d'abord présenté de nouvelles approches analytiques (électrophorétiques) applicables à diverses enzymes à potentiel antimicrobien. II a été aussi démontré que les chitinases, laminarinases et lysozymes d'origine végétale pouvaient se retrouver sous diverses formes dans divers organismes. Les plantes semblent plutôt riches en hydrolases fort diversifiées quant à leur forme moléculaire tandis que les chitosanases n'ont pas encore été rapportées chez les animaux. La signification de cette différence $n^{\prime}$ est pas encore élucidée. Pourtant, les animaux sont aussi exposés à des agents pathogènes appartenant aux Zygomycètes. Enfin, signalons que certaines activités (chitinase, chitosanase, laminarinase, lysozyme) peuvent se trouver sous la forme de protéines PR de stress et sous la forme de protéines constitutives dans des organes reproducteurs. Ce double rôle n'a pas été encore démontré chez les animaux. La signification de cette différence entre les plantes et les animaux n'est pas connue.

Au cours des prochaines années, l'étude des gènes et de l'expression de ces gènes dans des organismes transgéniques saura certes contribuer à mieux comprendre l'importance et I'utilisation de telles enzymes à potentiel microbien chez les divers types d'organismes. Enfin, cet article a voulu aussi signifier que l'expression monogénique de telles hydrolases dans des plants transgéniques ne constituait peut-être pas la panacée face aux agents pathogènes. Malgré cette limitation, nos connaissances sont encore trop embryonnaires pour bien exploiter tout le potentiel de ces enzymes à potentiel antimicrobien. 


\section{REMERCIEMENTS}

Les travaux de notre laboratoire rapportés dans cette revue de littérature ont été effectués grâce au soutien financier du Conseil de recherches en sciences naturelles et en génie (CRSNG), au Conseil des recherches en pêche et agro-alimentaire du Québec (CORPAQ) et au Fonds pour la formation des chercheurs et l'aide à la recherche (FCAR). Nous profitons de I'occasion pour remercier toutes les personnes qui ont contribué à ces travaux et à l'élaboration de ce texte.

\section{RÉFÉRENCES}

Anas, O., I. Alli et R.D. Reeleder. 1989. Inhibition of germination of sclerotia of Sclerotinia sclerotiorum by salivary gland secretions of Bradysia coprophila. Soil Biol. Biochem. 21: 47-52.

Araki, Y. et E. Ito. 1988. Chitin deacetylase. Pages 511-514 in W. A. Wood et S. T. Kellogg (réds.), Methods in enzymology. Vol. 161. Academic Press, San Diego, CA.

Audy, P. et A. Asselin. 1992. Gel electrophoretic analysis of chitosan hydrolysis products. Electrophoresis 13: 334-337.

Audy, P., J. Trudel et A. Asselin. 1988a. Purification and characterization of a lysozyme from wheat germ. Plant Sci. (Limerick) 58: 43-50.

Audy, P., N. Benhamou, J. Trudel et A. Asselin. 1988b. Immunocytochemical localization of a wheat germ lysozyme in wheat germ embryo and coleoptile cells and cytochemical study of its interaction with the cell wall. Plant Physiol. (Bethesda) 88: 1317-1322.

Audy, P., J. Grenier et A. Asselin. 1989. Lysozyme activity in animal extracts after sodium dodecyl sulfate-polyacrylamide gel electrophoresis. Comp. Biochem. Physiol. 92B: 523-527.

Audy, P., D. Lequéré, D. Leclerc et A. Asselin. 1990. Electrophoretic forms of Iysozyme activity in various plant species. Phytochemistry (Oxf.) 29: 1143-1159.

Ballance, G.M. et I. Svendsen. 1988. Purification and amino acid sequence determination of an endo-1,3-ß-glucanase from barley. Carlsberg Res. Commun. 53: 411-419.

Béliveau, C., C. Potvin, J. Trudel, A. Asselin et G. Bellemare. 1991. Cloning, sequencing and expression in Escherichia coli of a Streptococcus faecalis autolysin. J. Bacteriol. 173: 5619-5623.
Benhamou, N. et A. Asselin. 1989. Attempted localization of a substrate for chitinase in plant cells reveals abundant $\mathrm{N}$-acietyl-Dglucosamine residues in secondary walls. Biol. Cell 67: 341-350.

Benhamou, N., J. Grenier, A. Asselin et M. Legrand. 1989. Immunogold localization of $ß-1,3-$ glucanases in two plants infected by vascular wilt fungi. Plant Cell 1: 12091221.

Bjarnov, N. 1972. Carbohydrases in Chironomus, Gammarus and some Trichoptera larvae. Oikos 23: 261-263.

Blackwell, J. 1988. Physical methods for the determination of chitin structure and conformation. Pages 435-442 in W. A. Wood et S. T. Kellogg (réds.), Methods in enzymology. Vol. 161. Academic Press, San Diego, CA.

Bol, J.F., H.J.M. Linthorst et B.J.C. Cornelissen. 1990. Plant pathogenesis-related proteins induced by virus infection. Annu. Rev. Phytopathol. 28: 113-138.

Boller, T. 1988. Ethylene and the regulation of antifungal hydrolases in plants. Oxf. Surv. Plant Mol. Cell Biol. 5: 145-174.

Boman, H.G., I. Faye, G.H. Gudmundsson, J.Y. Lee et D.A. Lidholm. 1991. Cell-free immunity in Cecropia. A model system for antibacterial proteins. Eur. J. Biochem. 201: 23-31.

Broglie, K. , I. Chet, M. Holliday, R. Cressman, P. Biddle, S. Knowlton, C.J. Mauvals et R. Broglie. 1991. Transgenic plants with enhanced resistance to the fungal pathogen Rhizoctonia solani. Science (Wash. DC) 254: 1194-1197.

Bull, A.T. et C.G.C. Chesters. 1966. The biochemistry of laminarin and the nature of laminarinase. Adv. Enzymol. 28: 325-364.

Cabib, E. et B. Bowers. 1971. Chitin and yeast budding. Localization of chitin in yeast bud scars. J. Biol. Chem. 246: 152-159.

Cabib, E., R. Roberts et R. Bowers. 1982. Synthesis of the yeast cell wall and its regulation. Annu. Rev. Biochem. 51: 763793.

Chen, A.C., R.T. Mayer et V.R. Deloach. 1982. Purification and characterization of chitinase from the stable fly, Stomoxy's calcitrans. Arch. Biochem. Biophys. 216: 314321.

Chesters, C.G.C. et A.T. Bull. 1963. The enzymic degradation of laminarin. 1. The distribution of laminarinase among microorganisms. Biochem. J. 86: 28-46.

Cody, R.M. 1989. Distribution of chitinase and chitobiase in Bacillus. Curr. Microbiol. 19: 201-205. 
Côté, F., J. Letarte, J. Grenier, J. Trudel et A. Asselin. 1989. Detection of $ß-1,3-$ glucanase activity after native polyacrylamide gel electrophoresis: application to tobacco pathogenesis-related proteins. Electrophoresis 10: $527-529$

Côté, F., J. R. Cutt, A. Asselin et D.F. Klessig. 1991a. Pathogenesis-related acidic ß-1,3glucanase genes of tobacco are regulated by both stress and developmental signals. Mol. Plant-Microbe Interact. 4: 173-181.

Côté, F., S. El Ouakfaoui et A. Asselin. 1991b. Detection of $ß$-glucanase activity on various $ß-1,3-$ and $ß-1,4-$ glucans after native and denaturing polyacrylamide gel electrophoresis. Electrophoresis 12: 69-74.

Cutler, R.L. et D. Yellowlees. 1979. Purification and characterization of two endo-1,3ß-D-glucanases from Telescopium telescopium. Carbohydr. Res. 75: 221-229.

Dackman, C., I. Chet et B. Nordbring-Hertz. 1989. Fungal parasitism of the cyst nematode Heterodera schachtii: infection and enzymatic activity. FEMS (Fed. Eur. Microbiol. Soc.) Microbiol. Lett. 62: 201208.

De Bolle, M.F.C., I.J. Goderis, F.R.G. Teras, B.P.A. Cammue et W.F. Broekaert. 1991. A technique for detecting antifungal activity of proteins separated by polyacrylamide gel electrophoresis. Electrophoresis 12: 442-444.

Den Tandt, W.R., T. Inaba, I. Verhamme, B. Overdyk, V. Browwer et D. Prieur. 1988. Non-identity of human plasma lysozyme and 4-methylumbelliferyl-tetra- $\mathrm{N}$-acetylß-D-chitotetraoside hydrolase. Int. J. Biochem. 20: 713-719.

Dumas-Gaudot, E., J. Grenier, V. Furlan, J. Grenier et A. Asselin. 1992a. Chitinase, chitosanase and B-1,3-glucanase activities in Allium and Pisum roots colonized by Glomus species. Plant Sci. (Limerick) 84: 17-24.

Dumas-Gaudot, E., V. Furlan, J. Grenier et A Asselin. 1992b. New acidic chitinase isoforms induced in tobacco roots by vesicular-arbuscular mycorrhizal fungi. Mycorrhiza 1: 133-136.

El Ghaouth, A., J. Arul, J. Grenier et A. Asselin. 1991. Glucanohydrolases and inhibitory activity to Botrytis cinerea in extracts from strawberry fruits. Can. J. Plant Pathol. 13: 315-320.

El Ghaouth, A., J. Arul, J. Grenier et A. Asselin. 1992. Antifungal activity of chitosan on two postharvest pathogens on strawberry fruits. Phytopathology 82: 398-402.

El Ouakfaoui, S. et A. Asselin. 1992a. Multiple forms of chitosanase activities in plants. Phytochemistry (Oxf.) 31: 1513-1518.

El Ouakfaoui, S. et A. Asselin. 1992b. Diversity of chitosanase activity in cucumber. Plant Sci. (Limerick) 85: 33-41.
Fänge, R., G. Lundblad et J. Lind. 1976. Lysozyme and chitinase in blood and lymphomyeloid tissues of marine fish. Mar. Biol. (Berl.) 36: 277-282.

Fenouil, E. et P. Roch. 1991. Evidence and characterization of lysozyme in six species of freshwater crayfishes from Astacidae and Cambaridae families. Comp. Biochem. Physiol. 99B: 43-49.

Fenton, D.M. et D.E. Eveleigh. 1981. Purification and mode of action of a chitosanase from Penicillium islandicum. J. Gen. Microbiol. 126: 151-165.

Fink, W., M. Liefland et K. Mendgen. 1988. Chitinases and ß-1,3-glucanases in the apoplastic compartment of oat leaves (Avena sativa L.). Plant Physiol. (Bethesda) 88: 270-275.

Fuchs, R.L., S.A. McPherson et D.J. Drahos. 1986. Cloning of a Serratia marcescens gene encoding chitinase. Appl. Environ. Microbiol. 51: 504-509.

Funke, B. et K.D. Spindler. 1989. Characterization of chitinase from the brine shrimp Artemia. Comp. Biochem. Physiol. 94B: 691-695.

Grenier, J. et A. Asselin. 1990. Some pathogenesis-related proteins are chitosanases with lytic activity against fungal spores. Mol. Plant-Microbe Interact. 3: 401-407.

Grinde, B. 1989. A lysozyme from rainbow trout acts on mastitis pathogens. FEMS (Fed. Eur. Microbiol. Soc.) Microbiol. Lett. 60: $179-182$

Grinde, B., J. Jollès et P. Jollès. 1988. Purification and characterization of two lysozymes from rainbow trout (Salmo gairdneri). Eur. J. Biochem. 173: 269-273.

Grisley, M.S. et P.R. Boyle. 1990. Chitinase, a new enzyme in Octopus saliva. Comp. Biochem. Physiol. 95B: 311-316.

Hien, N.H. et G.H. Fleet. 1983. Separation and characterization of six 1,3-ß-glucanases from Saccharomyces cerevisiae. J. Bacteriol. 156: 1204-1213.

Hippe, S., K. Düring et F. Kreuzaler. 1989. In situ localization of a foreign protein in transgenic plants by immunoelectron microscopy following high pressure freezing. Freeze substitution and low temperature embedding. Eur. J. Cell Biol. 50: 230-234.

Hirano, S. 1988. Water-soluble glycol chitin and carboxymethylchitin. Pages $408-410$ in W. A. Wood et S. T. Kellogg (réds.), Methods in enzymology. Vol. 161. Academic Press, San Diego, CA.

Ibrahim, H.R., A. Kato et K. Kobayashi. 1991. Antimicrobial effects of lysozyme against gram-negative bacteria due to covalent binding of palmitic acid. J. Agric. Food Chem. 39: 2077-2082. 
Jollès, J., F. Schoentgen, G. Croizier, L. Croizier et P. Jollès. 1979. Insect lysozymes from three species of Lepidoptera: their structural relatedness to the c (chicken) type lysozyme. J. Mol. Evol. 14: 267-271.

Jollès, P. et J. Jollès. 1975. The lysozyme from Asterias rubens. Eur. J. Biochem. 54: 19-23.

Jollès, P. et J. Jollès. 1984. What's new in lysozyme research. Mol. Cell Biochem. 63: 165-189.

Kamei, K., Y. Yamamura, S. Hara et T. Ikenata. 1989. Amino acid sequence of chitinase from Streptomyces erythraeus. J. Biochem. 105: 979-985.

Kauffmann, S., M. Legrand, P. Geoffroy et B. Fritig. 1987. Biological function of "pathogenesis-related" proteins: four PR proteins of tobacco have 1,3-ß-glucanase activity. EMBO ( Eur. Mol. Biol. Organ.) J. 6: 32093212.

Kauss, H. et B. Bauch. 1988. Chitin deacetylase from Colletotrichum lindemuthianum. Pages 518-523 in W. A. Wood et S. T. Kellogg (réds.), Methods in enzymology. Vol. 161. Academic Press, San Diego, CA.

Kawamura, T. et G.D. Shockman. 1983. Purification and some properties of the endogenous, autolytic $\mathrm{N}$-acetylmuramoylhydrolase of Streptococcus faecium, a bacterial glycoenzyme. J. Biol. Chem. 258: 95149521.

Kitamura, K., T. Kaneko et Y. Yamamoto. 1971. Lysis of viable yeast cells by enzymes of Arthrobacter luteus. Arch. Biochem. Biophys. 145: 402-404.

Klebl, F. et W. Tanner. 1989. Molecular cloning of a cell wall exo-ß-1,3-glucanase from Saccharomyces cerevisiae. J. Bacteriol. 171: 6259-6264.

Knorr, D. 1986. Nutritional quality, food processing, and biotechnology aspects of chitin and chitosan: a review. Process Biochem. 21: 90-93.

Koga, D. et S. Kramer. 1983. Hydrolysis of glycol chitin by chitinolytic enzymes. Comp. Biochem. Physiol. 76B: 291-293.

Kono, M., T. Matsui, C. Shimizu et D. Koga. 1990. Purification and some properties of chitinase from the stomach of japanese eel, Anguilla japonica. Agric. Biol. Chem. 54: 973-978.

Kramer, K.J. et H. Aoki. 1987. Chitinolytic enzymes from pupae of the red flour beetle, Tribolium castaneum. Comp. Biochem. Physiol. 86B: 613-621.

Leclerc, D. et A. Asselin. 1989. Detection of bacterial cell wall hydrolases after denaturing polyacrylamide gel electrophoresis. Can. J. Microbiol. 35: 749-753.
Legrand, M., S. Kauffmann, P. Geoffroy et B. Fritig. 1987. Biological function of pathogenesis-related proteins: four tobacco pathogenesis-related proteins are chitinases. Proc. Nat. Acad. Sci. USA 84: 6750 6754.

Liénart, Y., J. Comtat et F. Barnoud. 1986. A wall-bound exo-1,3-ß-D-glucanase from Acacia cultured cells. Biochim. Biophys. Acta 883: 353-360.

Lotan, T. et R. Fluhr. 1990. Function and regulated accumulation of plant pathogenesis-related proteins. Symbiosis 8: 33-46.

Lotan, T., N. Ori et R. Fluhr. 1989. Pathogenesis-related proteins are developmentally regulated in tobacco flowers. Plant Cell 1: 881-887.

Lucas, J., A. Henschen, F. Lottspeich, U. Vögeli et T. Boller. 1985. Amino-terminal sequence of ethylene-induced bean leaf chitinase reveals similarities to sugar-binding domains of wheat germ agglutinin. FEBS (Fed Eur. Biochem. Soc.) Lett. 193: 208-210.

Lund, P. et P. Dunsmuir. 1992. A plant signal sequence enhances the secretion of bacterial Chi $A$ in transgenic tobacco. Plant Mol. Biol. 18: 47-53.

Lund, P., R.Y. Lee et P. Dunsmuir. 1989. Bacterial chitinase is modified and secreted in transgenic tobacco. Plant Physiol. (Bethesda) 91: 130-135.

Lundblad, G., B. Hederstedt, J. Lind et $M$. Steby. 1974. Chitinase in goat serum. Preliminary purification and characterization. Eur. J. Biochem. 46: 367-376.

Lyne, J.E., D.C. Carter, X-m He, G. Stubbs et J.H. Hash. 1990. Preliminary crystallographic examination of a novel fungal lysozyme from Chalaropsis. J. Biol. Chem. 265: 6928-6930.

Majeau, N., J. Trudel et A. Asselin. 1990. Diversity of cucumber chitinase isoforms and characterization of one seed basic chitinase with lysozyme activity. Plant Sci. (Limerick) 68: 9-16.

Manners, D.J. et G. Wilson. 1973. Studies on $ß$-glucanases. Some properties of a bacterial endo-ß-1,3-glucanase system. Biochem. J. 135: 11-18.

Manocha, M.S. et R. Balasubramanian. 1988. In vitro regulation of chitinase and chitin synthase activity of two mucoraceous hosts of a mycoparasite. Can. J. Microbiol. 34: 1116-1121.

Marshall, J.J. et R.J.A. Grand. 1975. Comparative studies on $ß$-glucan hydrolases. Isolation and characterization of an exo(1-3) ß-glucanase from the snail. Arch. Biochem. Biophys. 167: 165-175. 
Mauch, F., B. Mauch-Mani et T. Boller. 1988a. Antifungal hydrolases in pea tissue. I. Purification and characterization of two chitinases and two ß-1,3-glucanases differentially regulated during development and in response to fungal infection. Plant Physiol. (Bethesda) 88: 325-333.

Mauch, F., B. Mauch-Mani et T. Boller. 1988b. Antifungal hydrolases in pea tissue. II. Inhibition of fungal growth by combinations of chitinase and $ß-1,3-$ glucanase. Plant Physiol. (Bethesda) 88: 936-942.

McHenery, J.G., J.A. Allen et T.H. Birkbeck. 1986. Distribution of lysozyme-like activity in 30 bivalve species. Comp. Biochem. Physiol. 85B: 581-584.

Michia, J.C., G. Dandrifosse et C. Jeuniaux. 1973. Distribution et localisation tissulaire de la synthèse des chitinases chez les invertébrés inférieurs. Arch. Int. Physiol. Biochim. 81: 439-451.

Monaghan, R.L., D.E. Eveleigh, R.P. Tewari et E.T. Reese. 1973. Chitosanase, a novel enzyme. Nature New Biol. 245: 78-80.

Monreal, J. et E.T. Reese. 1968. The chitinase of Serratia marcescens. Can. J. Microbiol. 15: 689-696.

Nagel, R., R.G. Birch et J.M. Manners. 1990. Detection of bacterial chitinase activity in transformed plant tumour cells using a specific exochitinase substrate. Plant Cell Rep. 8: 729-732.

Nanjo, F., R. Katsumi et K. Sakai. 1991. Enzymatic method for determination of the degree of deacetylation of chitosan. Anal. Biochem. 193: 164-167.

Neuhaus, J. M., P. Ahl-goy, U. Hinz, S. Flores et F. Meinz. 1991a. High-level expression of a tobacco chitinase gene in Nicotiana sylvestris. Susceptibility of transgenic plants to Cercospora nicotianae infection. Plant Mol. Biol. 16: 141-151.

Neuhaus, J. M., L. Sticher, F. Meins et T. Boller. 1991b. A short C-terminal sequence is necessary and sufficient for the targeting of chitinases to the plant vacuole. Proc. Nat. Acad. Sci. USA 88: 10362-10366.

Novaes-Ledieu, M. et D. Garcia-Mendoza. 1981. The cells walls of Agaricus bisporus and Agaricus campestris fruiting body hyphae. Can. J. Microbiol. 27: 779-787.

Ohtakara, A., M. Izume et M. Mitsutomi. 1988. Action of microbial chitinases on chitosan with different degrees of deacetylation. Agric. Biol. Chem. 52: 3181-3182.

Pelletier, A. et J. Sygusch. 1990. Purification and characterization of three chitosanase activities from Baccillus megaterium P1. Appl. Environ. Microbiol. 56: 844-848.

Perry, L.J. et R. Whetzel. 1984. Disulfide bond engineered into T4 lysozyme: stabilization of the protein toward thermal inactivation. Science (Wash. DC) 226: 555-557.
Perry, L.J. et R. Whetzel. 1986. Unpaired cysteine-54 interferes with the ability of an engineered disulfide to stabilize T4 lysozyme. Biochemistry 25: 733-739.

Pont Lezica, R. et L. Quesada-Allué. 1990. Chitin. Pages 443-481 in D. M. Dey et J. B. Harborne (réds.), Methods in plant biochemistry. Vol. 2. Academic Press, San Diego, CA.

Potvin, C., D. Leclerc, G. Tremblay, A. Asselin et G. Bellemare. 1988. Cloning, sequencing and expression of a Bacillus bacteriolytic enzyme in Escherichia coli. Mol. \& Gen. Genet. 214: 241-248.

Reyes, F., R. Lahoz, M.J. Martinez et C. Alfonso. 1985. Chitosanases in the autolysis of Mucor rouxii. Mycopathologia 89: 181-187.

Reyes, F., J. Calatayud et M.J. Martinez. 1988. Chitinolytic activity in the autolysis of Aspergillus nidulans. FEMS (Fed. Eur. Microbiol. Soc.) Microbiol. Lett. 49: 239243.

Robbins, P.W., C. Albright et B. Benfield. 1988. Cloning and expression of a Streptomyces plicatus chitinase (chitinase-63) in Escherichia coli. J. Biol. Chem. 263: 443-447.

Rombouts, F.M. et H.J. Phaff. 1976. Lysis of yeast cell walls. Lytic ß-1,3-glucanases from Bacillus circulans WL-12. Eur. J. Biochem. 63: 121-130.

Rossignol, P.A. et A.M. Lueders. 1986. Bacteriolytic factor in the salivary glands of Aedes aegypti. Comp. Biochem. Physiol. 83B: 819822.

Saedi, M.S., K.J. Garvey et J. Ito. 1987. Cloning and purification of a unique lysozyme produced by Bacillus phage $\varphi 29$. Proc. Nat. Acad. Sci. USA 84: 955-958.

Sakellaris, H., J.M. Pemberton et J.M. Manners. 1990. Genes from Cellvibrio mixtus encoding ß-1,3-endoglucanase. Appl. Environ. Microbiol. 56: 3204-3208.

Samac, D.A. et D.M. Shah. 1991. Developmental and pathogen-induced activation of the Arabidopsis acidic chitinase promoter. Plant Cell 3: 1063-1072.

Schlumbaum, A., F. Mauch, U. Vögeli et T. Boller. 1986. Plant chitinases are potent inhibitors of fungal growth. Nature (Lond.) 324: 365-367.

Schneider, P.M. 1985. Purification and properties of three lysozymes from haemolymph of the cricket, Gryllus bimaculatus (De Geer). Insect Biochem. 15: 463-470.

Shapira, R., A. Ordentlich, I. Chet et A.B. Oppenheim. 1989. Control of plant diseases by chitinase expressed from cloned DNA in Escherichia coli. Phytopathology 79: 1246-1249. 
Shinshi, H., J.M. Neuhaus, J. Ryals et F. Meins Jr. 1990. Structure of a tobacco endochitinase gene: evidence that different chitinase genes can arise by transposition of sequences encoding a cysteine-rich domain. Plant Mol. Biol. 14: 357-368.

Smucker, R.A. et D.A. Wright. 1986. Characteristics of Crassostrea virginica crystalline style chitin digestion. Comp. Biochem. Physiol. 83A: 489-493.

Spies, A.G., J.E. Karlinsey et K.D. Spence. 1986. Antibacterial hemolymph proteins of Manduca sexta. Comp. Biochem. Physiol. 83B: 125-133.

Srivastava, A.K., G. Défago et T. Boller. 1985. Secretion of chitinase by Aphanocladium album, a hyperparasite of wheat rust. Experientia (Basel) 41: 1612-1613.

St-Leger, R.J., R.M. Cooper et A.K. Charnley. 1986a. Cuticle-degrading enzymes of entomopathogenic fungi: regulation of production of chitinolytic enzymes. J. Gen. Microbiol. 132: 1509-1517.

St-Leger, R.J., R.M. Cooper et A.K. Charnley. 1986b. Cuticle-degrading enzymes of entomopathogenic fungi: cuticle degradation in vitro by enzymes from entomopathogens. J. Invertebr. Pathol. 47: 167-177.

Stanford, A., M. Bevan et D. Northcote. 1989. Differential expression within a family of novel wound induced genes in potato. Mol. \& Gen. Genet. 215: 200-208.

Steinert, S.A. et G.V. Pickwell. 1984. Quantitative determination of Iysozyme in the hemolymph of Mytilus edulis by the enzyme-linked immunosorbent assay (ELISA). Mar. Environ. Res. 14: 229-243.

Sundheim, L., A.R. Poplawsky et A.H. Ellingboe. 1988. Molecular cloning of two chitinase genes from Serratia marcescens and their expression in Pseudomonas species. Physiol. Mol. Plant Pathol. 33: 483-491.

Takeda, H., G.A. Strasdine, D.R. Whitaker et C. Roy. 1966. Lytic enzymes in the digestive juice of Helix pomatia. Chitinases and muramidases. Can. J. Biochem. 44: 509518.

Taniyama, Y., R. Kuroki, F. Omura, C. Seko et M. Kikuchi. 1991. Evidence for intramolecular disulfide bond shuffling in the folding of mutant human lysozyme. J. Biol. Chem. 266: 6456-6461.
Trudel, J. et A. Asselin. 1989. Detection of chitinase activity after polyacrylamide gel electrophoresis. Anal. Biochem. 178: 362366.

Trudel, J. et A. Asselin. 1990. Detection of chitin deacetylase activity after polyacrylamide gel electrophoresis. Anal. Biochem. 189: 249-253.

Trudel, J., P. Audy et A. Asselin. 1989. Electrophoretic forms of chitinase activity in Xanthi-nc tobacco, healthy and infected with tobacco mosaic virus. Mol. PlantMicrobe Interact. 2: 315-324.

Trudel, J., C. Potvin et A. Asselin. 1992. Expression of active hen egg white lysozyme in transgenic tobacco. Plant Sci. (Limerick) 87: 55-67.

Truschel, M.R., S.A. Chambers et D.R. McLay. 1986. Two antigenically distinct forms of ß-1,3-glucanase in sea urchin embryonic development. Dev. Biol. 117: 277-285.

Upadhyay, J.M., B.A. Mares, D.M. Hennelt et P.G. Rivet. 1977. Purification and specificity of bacteriolytic enzyme 1 from Hartmannella glebae. Appl. Environ. Microbiol. 33: $1-5$.

Vigers, A.J., W.K. Roberts et C.P. Selitrennikoff. 1991. A new family of plant antifungal proteins. Mol. Plant-Microbe Interact. 4: $315-323$.

Ward, E.R., S.V. Uknes, S.C. Williams, S.S. Dincher, D.L. Wiederhold, D.C. Alexander, P. Ahl-Goy, J.P. Métraux et J.A. Ryals. 1991. Coordinate gene activity in response to agents that induce systemic acquired resistance. Plant Cell 3: 1085-1094.

Ward, K.A. et D. Fairbairn. 1972. Chitinase in developing eggs of Ascaris suum (nematoda). J. Parasitol. 58: 546-549.

White, R.F. et J.F. Antoniw. 1991. Virus-induced resistance responses in plants. Crit. Rev. Plant Sci. 9: 443-455.

Wong, Y.-S. et G.A. Maclachlan. 1980. 1,3-ßD-glucanases from Pisum sativum seedlings. III. Development and distribution of endogenous substrates. Plant Physiol. (Bethesda) 65: 222-228. 